\title{
Genetic Interactions Explain Variance in Cingulate Amyloid Burden: An AV-45 PET Genome-Wide Association and Interaction Study in the ADNI Cohort
}

\author{
Jin Li, ${ }^{1,2}$ Qiushi Zhang, ${ }^{1,2,3}$ Feng Chen, ${ }^{1}$ Jingwen Yan, ${ }^{4,5,6}$ Sungeun Kim, ${ }^{4}$ Lei Wang, ${ }^{1,2}$ \\ Weixing Feng, ${ }^{1,2}$ Andrew J. Saykin, ${ }^{4}$ Hong Liang, ${ }^{1,2,4}$ and Li Shen ${ }^{4,5,6}$ \\ ${ }^{1}$ Institute of Biomedical Engineering, College of Automation, Harbin Engineering University, 145 Nantong Street, Harbin 150001, China \\ ${ }^{2}$ Center for Bioinformatics, College of Automation, Harbin Engineering University, 145 Nantong Street, Harbin 150001, China \\ ${ }^{3}$ College of Information Engineering, Northeast Dianli University, 169 Changchun Street, Jilin 132012, China \\ ${ }^{4}$ Center for Neuroimaging, Department of Radiology and Imaging Sciences, Indiana University School of Medicine, \\ 355 West 16th Street, Suite 4100, Indianapolis, IN 46202, USA \\ ${ }^{5}$ Center for Computational Biology and Bioinformatics, Indiana University School of Medicine, 410 West 10th Street, \\ Suite 5000, Indianapolis, IN 46202, USA \\ ${ }^{6}$ Department of Biohealth Informatics, Indiana University School of Informatics and Computing, Indianapolis, IN 46202, USA
}

Correspondence should be addressed to Hong Liang; liangh@iu.edu and Li Shen; shenli@iu.edu

Received 15 December 2014; Accepted 17 March 2015

Academic Editor: Dongchun Liang

Copyright (C) 2015 Jin Li et al. This is an open access article distributed under the Creative Commons Attribution License, which permits unrestricted use, distribution, and reproduction in any medium, provided the original work is properly cited.

\begin{abstract}
Alzheimer's disease $(\mathrm{AD})$ is the most common neurodegenerative disorder. Using discrete disease status as the phenotype and computing statistics at the single marker level may not be able to address the underlying biological interactions that contribute to disease mechanism and may contribute to the issue of "missing heritability." We performed a genome-wide association study (GWAS) and a genome-wide interaction study (GWIS) of an amyloid imaging phenotype, using the data from Alzheimer's Disease Neuroimaging Initiative. We investigated the genetic main effects and interaction effects on cingulate amyloid-beta (A $\beta)$ load in an effort to better understand the genetic etiology of $\mathrm{A} \beta$ deposition that is a widely studied $\mathrm{AD}$ biomarker. PLINK was used in the single marker GWAS, and INTERSNP was used to perform the two-marker GWIS, focusing only on SNPs with $p \leq 0.01$ for the GWAS analysis. Age, sex, and diagnosis were used as covariates in both analyses. Corrected $p$ values using the Bonferroni method were reported. The GWAS analysis revealed significant hits within or proximal to APOE, APOC1, and TOMM40 genes, which were previously implicated in AD. The GWIS analysis yielded 8 novel SNP-SNP interaction findings that warrant replication and further investigation.
\end{abstract}

\section{Introduction}

Alzheimer's disease (AD) is the most common neurodegenerative disorder characterized by a progressive decline in memory and cognition. The pathologic cascade in $\mathrm{AD}$ involves two primary hallmarks: amyloid- $\beta(\mathrm{A} \beta)$ plaques and neurofibrillary tangles [1]. Genetics plays an important role in late-onset Alzheimer's disease (LOAD), but missing heritability remains to be found according to current approximations
[2]. The last several decades of research yielded only one genetic risk factor of large effect for LOAD: Apolipoprotein $\mathrm{E}$ (APOE) with 2 copies of the $\varepsilon 4$ allele confers approximately 6 to 30 -fold risk for the disease [3]. Some recent genome-wide association studies (GWAS) have identified several additional AD susceptibility genes, including BIN1, CLU, ABCA7, CR1, PICALM, MS4A6A, MS4A4E, CD33, CD2AP, and EPHA1 [49]. However, these genetic factors have relatively low effect sizes (odds ratios of 0.87-1.23) and cumulatively account for 
approximately 35\% of population-attributable risk [8]. More recently, a large scale GWAS meta-analysis identified 11 new susceptibility loci with also small effect sizes [10].

Traditional GWAS analyses used discrete disease status as the phenotypic trait of interest despite the fact that LOAD is a clinically heterogeneous disorder. Recently, researchers started to explore intermediate quantitative traits (QTs), such as clinical or cognitive features, biochemical assays, or neuroimaging biomarkers, in genetic association testing. This may have the potential to address the issue of clinical heterogeneity in LOAD. These QTs are often measured as continuous variables and thus exhibit a higher genetic signalto-noise ratio. Further, most intermediate QTs are more proximal to their genetic bases than disease status. Thus, the incorporation of intermediate QTs can potentially increase statistical power to detect disease-related genetic associations $[11,12]$. An ancillary benefit of using QTs is that they can serve as effective biomarkers for monitoring disease progress or treatment response in clinical practice or drug trials.

Over the past 10-15 years, studies have identified robust and predictive biomarkers for AD including levels of tau and amyloid- $\beta$ peptides in cerebrospinal fluid (CSF), selective measures of brain atrophy using magnetic resonance imaging (MRI), and imaging of glucose hypometabolism and amyloid using positron emission tomography (PET) [13]. PET imaging can be used to quantify levels of amyloid in the brain by utilizing a radiotracer such as florbetapir $\left({ }^{18} \mathrm{~F}-\mathrm{AV}-45\right.$ or AV-45) or/and Pittsburgh compound-B (PiB, N-methyl$\left[{ }^{11} \mathrm{C}\right]^{2}$-(40-methylaminophenyl)-6-hydroxybenzothiazole). These amyloid measures have been studied as biomarkers for classifying AD [14-17]. All these multimodal biomarkers can potentially be served as AD relevant QTs and have been examined in many existing quantitative genetics studies of LOAD [18].

In addition, most genetic association studies compute statistics at the single marker level and ignore the possible underlying biological interactions that contribute to the development of disease [19] and could be a possible source for "missing heritability." Given the quadratically growing search space of two-way interactions, we are facing major computational and statistical challenges. To address this issue, one approach is to effectively explore epistatic interactions in genome-wide data by using a priori statistical and/or biological evidence to generate a reduced set of genetic markers for interaction testing. Using this strategy, previous interaction studies in LOAD (e.g., [20-24]) implicated interactions between $C R 1$ and $A P O E$ using quantified $\mathrm{A} \beta$ PET as the outcome variable [24] and between cholesterol trafficking genes [21, 22] and tau phosphorylation genes [20] in case-control analyses. These studies demonstrated that the important information could be garnered from investigating genetic interactions in complex diseases like LOAD.

With these observations, in the present work, we conducted a quantitative genetics study of an $\mathrm{AD}$-associated amyloid imaging phenotype and examined both single marker main effects and two-marker interaction effects at the genome-wide level. Specifically, we investigated the main and interaction effects of genome-wide markers on cingulate amyloid-beta $(\mathrm{A} \beta)$ load in an effort to better understand the genetic etiology of cingulate cortical $\mathrm{A} \beta$ deposition (a LOAD biomarker).

\section{Materials and Methods}

Data used in the preparation of this paper were obtained from the Alzheimer's Disease Neuroimaging Initiative (ADNI) database (http://adni.loni.usc.edu/). The ADNI was launched in 2003 by the National Institute on Aging (NIA), the National Institute of Biomedical Imaging and Bioengineering (NIBIB), the Food and Drug Administration (FDA), private pharmaceutical companies, and nonprofit organizations, as a $\$ 60$ million, 5-year public-private partnership. The primary goal of ADNI has been to test whether serial magnetic resonance imaging (MRI), positron emission tomography (PET), other biological markers, and clinical and neuropsychological assessment can be combined to measure the progression of mild cognitive impairment (MCI) and early Alzheimer's disease (AD). Determination of sensitive and specific markers of very early $\mathrm{AD}$ progression is intended to aid researchers and clinicians to develop new treatments and monitor their effectiveness, as well as lessen the time and cost of clinical trials.

The Principal Investigator of this initiative is Michael W. Weiner, M.D., VA Medical Center and University of California, San Francisco. ADNI is the result of efforts of many coinvestigators from a broad range of academic institutions and private corporations, and subjects have been recruited from over 50 sites across the US and Canada. The initial goal of ADNI was to recruit 800 subjects but ADNI has been followed by ADNI-GO and ADNI-2. To date these three protocols have recruited over 1500 adults, aged 55 to 90, to participate in the research, consisting of cognitively normal older individuals, people with early or late MCI, and people with early AD. The follow-up duration of each group is specified in the protocols for ADNI-1, ADNI-2, and ADNIGO. Subjects originally recruited for ADNI-1 and ADNI-GO had the option to be followed in ADNI-2. For up-to-date information, see http://www.adni-info.org/.

We applied for and were granted permission to use data from the ADNI cohort (http://www.adni-info.org/) to conduct genetic association and interaction analyses.

2.1. Subjects and Data. For the present work, analyses were restricted to subjects with both genotyping data and AV-45 PET data available. The study sample $(N=602)$ included 190 healthy control (HC), 215 early MCI (EMCI), 152 late MCI (LMCI), and 45 AD subjects. Table 1 shows selected demographic and clinical characteristics of these participants at the time of the baseline AV-45 PET scan.

2.2. Genotyping Data and Quality Control. The genotyping data of the participants were collected using either the Illumina $2.5 \mathrm{M}$ array (a byproduct of the ADNI whole genome sequencing sample) or the Illumina OmniQuad array [18, 25, 26]. For the present analyses, we included single nucleotide 
polymorphism (SNP) markers that were present on both arrays.

Quality control (QC) was performed using the PLINK software (version 1.07) [27]. SNPs not meeting any of the following criteria were excluded from further analyses: (1) call rate per SNP $\geq 95 \%$; (2) minor allele frequency $\geq 5 \%(n=117$, 175 SNPs were excluded based on criteria 1 and 2); and (3) Hardy-Weinberg equilibrium test of $p \geq 10^{-6}(n=997$ SNPs were excluded) using control subjects only. Participants were excluded from the analysis if any of the following criteria were not satisfied: (1) call rate per participant $\geq 90 \%$ (3 participants were excluded); (2) sex check (1 participant was excluded); and (3) identity check for related pairs (3 sibling pairs were identified with PI_HAT $>0.5$; one participant of each pair was randomly selected and excluded from the study).

Population stratification analysis was performed using EIGENSTRAT [28] and confirmed using STRUCTURE [29]. It yielded 47 study participants who did not cluster with the remaining subjects and with the CEU HapMap samples who are primarily of European ancestry (non-Hispanic Caucasians). These 47 participants were excluded from the analysis. After QC, 582,718 SNPs and 602 samples remained available for genetic association and interaction analyses.

2.3. Quantitative Traits. A previous AV-45 PET study [30] showed that both $\mathrm{AD}$ and amnestic MCI subjects had higher standardized uptake value ratio (SUVR) in global cortical, precuneus, frontal, occipital, and posterior cingulate areas. We focused this study in one of these regions, which is cingulate. UC Berkeley extracted baseline SUVR mean measure from the cingulate cortical region (version 2014.7.30) that was downloaded from the ADNI database (http://adni.loni.usc.edu/) for 987 ADNI-GO/2 participants. We also downloaded the cerebellum SUVR measure and used it to normalize the cingulate SUVR measure. The normalized SUVR was used as the quantitative trait (QT) in our analyses. After excluding 383 participants due to the lack of genotyping data, 602 individuals remained in the further analysis.

In addition, amyloid- $\beta 1-42$ peptide ( $\mathrm{A} \beta-42)$, total tau ( $\mathrm{t}-$ tau), and tau phosphorylated at the threonine 181 (p-tau181p), measured in CSF samples, are potential diagnostic biomarkers for AD [31-33]. Among the 602 individuals, 504 have both AV-45 data and CSF data. Following a previous GWAS study on CSF biomarkers [34], QC was performed on the CSF data to reduce the potential influence of extreme outliers on statistical results. Mean and standard deviation (SD) of Aß142 and 2 ratios (t-tau/Aß1-42 and p-taul81p/Aß1-42) were calculated, blind to diagnostic information. Subjects who had at least one value greater or smaller than 4 SDs from the mean value of each of 3 CSF variables were regarded as extreme outliers and removed from the analysis. This step removed 5 additional participants, resulting in 499 valid CSF samples.

2.4. Genetic Association Studies: Main Effects and Interaction Effects. For GWAS examining the main effects, linear regression was performed using PLINK to determine the association of each SNP to the AV-45 measure. An additive genetic model was tested with covariates including age, gender, and diagnosis (through four binary dummy variables indicating HC, EMCI, LMCI, or AD). Manhattan plots and Q-Q plots were generated using Haploview (http://www .broad.mit.edu/mpg/haploview/) and R (http://www.r-project.org/), respectively.

For GWIS examining the interaction effects, the INTERSNP software [35] was applied to the genotyping data and phenotypic AV- 45 measure. First, a single marker $p$ value for the main effect was computed for each SNP. Top 10,000 SNPs with the smallest $p$ values were selected and included in the subsequent interaction analysis. An explicit test for additive interaction (the full model including both additive and dominance effects plus interaction term versus reduced model that does not contain interaction terms) was performed on all possible SNP pairs among the top 10,000 SNPs, using twomarker analysis. The computation was conducted in a linear regression framework. We examined the association between SNP-SNP interactions and the AV-45 measure while controlling for relevant covariates at the baseline scan, including age, sex, and clinical diagnosis. This resulted in a total of approximately 50 million unique SNP pairs to be tested from the ADNI dataset. Interactions were considered significant if their Bonferroni corrected $p$ value $<0.05$.

2.5. Post Hoc Analysis. For identified significant interactions, we applied hierarchical linear regression using IBM SPSS 20 to estimate the amount of variance $\left(R^{2}\right)$ in the $\mathrm{AV}-45$ measure accounted for by these interaction terms. We first included the same set of covariates (age, gender, and diagnosis) in the linear model. After that, we included $A P O E$ status, the closest SNP to the BCHE SNP identified in a prior amyloid GWAS study [36], and the two SNP main effects from the identified SNP pair. Finally, we included the SNP-SNP interaction term to calculate additional variance explained by the interaction term. The difference in $R^{2}$ for the significant models was calculated in SPSS as $\Delta R^{2}=R^{2}$ (full model with interaction term) $-R^{2}$ (reduced model without interaction term). Significant effects were plotted in SPSS as well.

In addition, based on the identified interactions associated with $\mathrm{AV}-45$, we further evaluated their main and interaction effects on the CSF levels related to amyloid, including $\mathrm{A} \beta 1-42, \mathrm{t}$-tau181p/A $\beta 1-42$, and $\mathrm{p}$-tau/A $\beta 1-42$. These three CSF measures were used as the QTs in 3 separate genetic analyses, following the same method and steps for analyzing $\mathrm{AV}-45$ phenotype as described above.

\section{Results and Discussion}

3.1. GWAS Results. Table 1 shows selected demographic and clinical characteristics of 602 ADNI participants analyzed in this study, where the EMCI group is slightly younger than the other groups. Figure 1 shows the Q-Q plot, indicating no evidence of spurious inflation. Figure 2 shows the Manhattan plot. As expected, significant associations were identified between loci on chromosome 19 and the AV-45 measure. The top association is from $\mathrm{rs} 4420638\left(P=5.11 \times 10^{-21}\right)$, which codes for the APOC1 [37]. A few other SNPs within the 
TABLE 1: Selected demographic and clinical characteristics of participants at the time of AV-45 PET scan.

\begin{tabular}{|c|c|c|c|c|}
\hline & $\mathrm{HC}(N=190)$ & $\operatorname{EMCI}(N=215)$ & LMCI $(N=152)$ & $\mathrm{AD}(N=45)$ \\
\hline Age (years) & $74.51(5.74)$ & $71.43(7.28)$ & $73.03(7.49)$ & $74.87(9.05)$ \\
\hline Women & $94(49 \%)$ & $95(44 \%)$ & $62(41 \%)$ & $17(38 \%)$ \\
\hline Education (years) & $16.53(2.64)$ & $15.95(2.66)$ & $16.32(2.90)$ & $15.67(2.70)$ \\
\hline$A P O E$ e 4 allele present & $54(28 \%)$ & $87(40 \%)$ & $78(51 \%)$ & $33(73 \%)$ \\
\hline CDR-SOB & $0.03(0.13)$ & $1.22(0.72)$ & $1.73(0.94)$ & $4.36(1.64)$ \\
\hline Mini mental status examination & $29.07(1.20)$ & $28.39(1.46)$ & $27.25(1.77)$ & $22.93(2.08)$ \\
\hline Logical memory immediate recall (WMS-R) & $14.46(3.08)$ & $10.96(2.77)$ & $7.32(3.06)$ & $4.40(2.52)$ \\
\hline Logical memory delayed recall (WMS-R) & $13.55(3.27)$ & $8.90(1.72)$ & $4.22(2.75)$ & $2.02(2.17)$ \\
\hline Normalized SUVR of cingulate amyloid burden & $1.211(0.21)$ & $1.273(0.23)$ & $1.274(0.27)$ & $1.48(0.24)$ \\
\hline
\end{tabular}

AD: Alzheimer's disease; CDR-SOB: clinical dementia rating-sum of boxes; EMCI: early mild cognitive impairment; HC: healthy control; LMCI: late mild cognitive impairment; PET: positron emission tomography; WMS-R: Wechsler Memory Scale-Revised. Data are shown in the format of "number (\%)" or "mean (SD)."

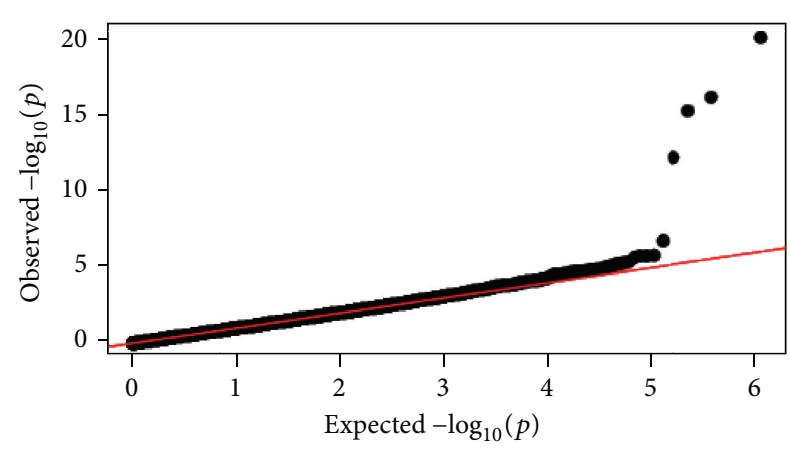

FIGURE 1: Quantile-quantile (Q-Q) plot of the observed - $\log _{10} p$ values from the GWAS of cingulate cortical $A ß$ load versus those expected under the null hypothesis.

$A P O E$ region, including adjacent $A P O C 1$ and TOMM40, were significantly associated with the AV-45 level in cingulate.

3.2. SNP-SNP Interaction Results. The INTERSNP model we tested included age, sex, and diagnosis as covariates. Eight SNP pairs showed significant interaction effects on the cingulate AV45 measure (corrected $p$ value < 0.05) (Table 2): rs2194938 (CLSTN2)-rs7644138 (FHIT), rs7916162 (TACC2)-rs2326536 $\left(P R N P^{*}\right), \quad$ rs2295873 (TACC2)-rs7794838 (IGFBP3*), $\quad$ rs2295874 (TACC2)rs2326536 $\left(P R N P^{*}\right)$, rs13056151 (BCR)-rs17594541 (MAGI2), rs13426621 (LOC388942)-rs7037332 (TYRP1* $\left.{ }^{*}\right)$, rs16936424 (LOC387761)-rs10504164 (N/A), and rs16939265 (HNF4G ${ }^{*}$ )rs6854047 (RWDD4*).

3.3. Post Hoc Analysis. Table 2 also shows the results of post hoc analysis on cingulate amyloid deposition. Age, gender, and diagnosis were first included in the model and accounted for $11 \%$ of variance in the amyloid QT. APOE status was then accounted for an additional $16.1 \%$ of variance, followed by the closest SNP to the BCHE SNP identified in [36] accounted for an additional $1.8 \%$ of variance. For each interaction, we ran a hierarchical linear regression model. We first added in the genetic main effects and then the genetic interaction term to determine the variance associated with the interaction term alone. For rs2194938 (CLSTN2)-rs7644138 (FHIT), the SNP main effects accounted for $3.4 \%$ of variance, and the interaction term accounted for $4.9 \%$ of variance ( $8.3 \%$ combined). For rs7916162 (TACC2)-rs2326536 $\left(P R N P^{*}\right)$, the main effects accounted for $2 \%$ of variance, and the interaction accounted for $4.9 \%$ of variance $(6.9 \%$ combined). For rs2295873 (TACC2)-rs7794838 (IGFBP $\left.{ }^{*}\right)$, the main effects accounted for $3.7 \%$ of variance, and the interaction term accounted for $4.1 \%$ of variance $(7.8 \%$ combined). For rs2295874 (TACC2)rs2326536 (PRNP* ${ }^{*}$, the SNP main effects accounted for $3.7 \%$ of variance, and the interaction term accounted for $4.1 \%$ of variance (7.8\% combined). For rs13056151 (BCR)-rs17594541 (MAGI2), the main effects accounted for $3.5 \%$ of variance, and the interaction term accounted for $2.6 \%$ of variance (6.1\% combined). For rs13426621 (LOC388942)-rs7037332 $\left(T Y R P 1^{*}\right)$, the main effects accounted for $4.2 \%$ of variance, and the interaction accounted for $2.3 \%$ of variance $(6.5 \%$ combined). For rs16936424 (LOC387761)-rs10504164 (N/A), the main effects accounted for $3.7 \%$ of variance, and the interaction term accounted for $1.7 \%$ of variance (5.4\% combined). For rs16939265 (HNF4G*)-rs6854047 (RWDD4*), the main effects accounted for $2.7 \%$ of variance, and the interaction term accounted for $1.3 \%$ of variance ( $4.0 \%$ combined).

Using a slightly reduced sample $(N=499)$ with CSF biomarker data available, all 8 identified interactions remained statistically significant when performing hierarchical linear regression using the CSF phenotypes (one baseline measure: $\mathrm{A} \beta$, two ratios: $\mathrm{t}-\mathrm{Tau} / \mathrm{A} \beta$ and $\mathrm{p}-\mathrm{Tau} / \mathrm{A} \beta$ ) instead of the AV-45 measure as outlined earlier (Table 3). We also repeated the same AV-45 analysis on the reduced sample and achieved a very similar result (Table 4 ).

3.4. Discussion. In this study, we performed both GWAS and GWIS analyses of the cingulate AV-45 florbetapir PET measure, using a sample of 602 subjects from the ADNI database. To our knowledge, this is the first genome-wide study on examining SNP-SNP interaction effects on cingulate amyloid deposition in a substantially large sample. In the single marker analysis, as expected, SNPs in APOE, APOC1, and TOMM40 genes (Figure 2) exhibited genome-wide 


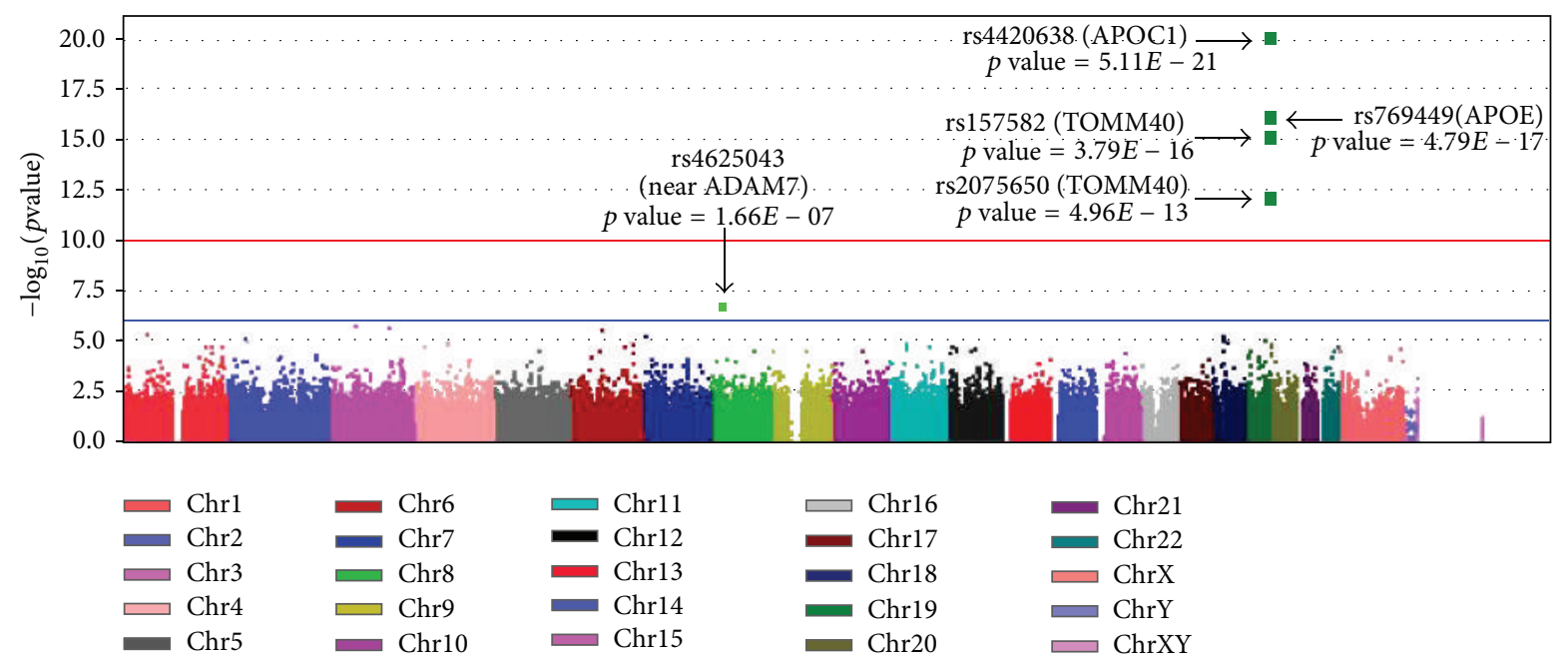

FIGURE 2: Manhattan plot of the observed $-\log _{10} p$ values from the GWAS of cingulate cortical A $\beta$ load. More than 580,000 SNPs were tested for association with cingulate cortical A $\beta$ burden under an additive model, with age, gender, and diagnosis as covariates. Genome-wide significant associations (exceeding the threshold represented by the red line and determined by Bonferroni correction) were identified on chromosome 19 within the $A P O E$ and its neighboring regions.

significant associations to the cingulate cortical Aß level. Two-marker interaction analyses revealed 8 SNP pairs, which had significant genetic interactions (corrected $p \leq 0.05)$ with cingulate amyloid burden. The risk variants at these pairs had low main effects but explained a relatively high-level variance of the amyloid deposition in cingulate (Table 2).

In addition, missing heritability can partially be explained by the interaction effects that are not examined in traditional GWAS analyses. Genetic risk underlying diagnosis of LOAD is considered to be manifested from multiple genes which interact with each other. We have performed a post hoc analysis investigating the effects of the identified SNP-SNP interactions LOAD related quantitative phenotypes including amyloid deposition and CSF biomarkers $(\mathrm{A} \beta, \mathrm{t}-\mathrm{tau} / \mathrm{A} \beta, \mathrm{p}-$ $\operatorname{tau} / \mathrm{A} \beta)$. Given amyloid and tau phosphorylation as major $\mathrm{AD}$ hallmarks, it is not surprising to observe the genetic interaction effects on both the amyloid load and relevant CSF biomarkers (Tables 2-4). Our results suggest that significant SNP-SNP interactions could exist between SNPs with low and insignificant main effects, and these interactions could be associated with altered amyloid burden and explain highlevel risk in AD.

In line with our hypothesis, we identified multiple significant genetic interactions associated with cingulate amyloid deposition. Several genes found in this study have already been implicated in $\mathrm{AD}$, thus lending confidence to the analytic procedure and results. These genes include PRNP [38, 39], IGFBP3 [40, 41], and MAGI2 [42, 43]. For example, Guerreiro et al. reported a nonsense mutation in PRNP associated with clinical Alzheimer's disease [38]. Ikonen et al. showed that interaction between the Alzheimer's survival peptide humanin and insulin-like growth factor-binding protein $3(I G F B P 3)$ regulates cell survival and apoptosis
[40]. Potkin et al. identified an MAGI2 SNP associated with hippocampal atrophy using the ADNI data [42]. Perhaps more importantly, this study also identified a number of SNPs that had not yet been associated with $\mathrm{AD}$ in conventional GWAS studies. Thus, this study exposes several potential candidate genes that could be explored in future replication samples.

This study had several methodological and technical advantages over other imaging genetics studies in addition to the above interesting findings. (1) To our knowledge this is the first genome-wide study to explore how SNP-SNP interactions influence cingulate amyloid burden, measured using florbetapir PET scan information. (2) Using continuous quantitative traits as phenotypes confers higher statistical power than using conventional clinical status. (3) The sample in this study included HC, EMCI, LMCI, and AD, thus providing a continuous and wide spectrum of the disease progression in the dataset. (4) Our approach embraced, rather than ignored, the confounding factors including age, sex, diagnosis, and previously identified risk genes $A P O E$ and $B C H E$ and provided more accurate estimate of the interaction effects on amyloid burden. (5) CSF data were used in this study to cross-check the identified interactions, which had the potential to serve as an indirect validation strategy or provide complemental information.

Our study has several limitations. (1) We used single marker main effect value to select SNPs for interaction analysis, which could miss significant interactions between SNPs with insignificant main effects. (2) The small cell size in the interaction analyses might introduce false positives. (3) Our approach is mostly data-driven, without utilizing any existing biological knowledge (e.g., pathways, networks, and other functional annotation data), which may reduce the statistical power and result interpretability. 


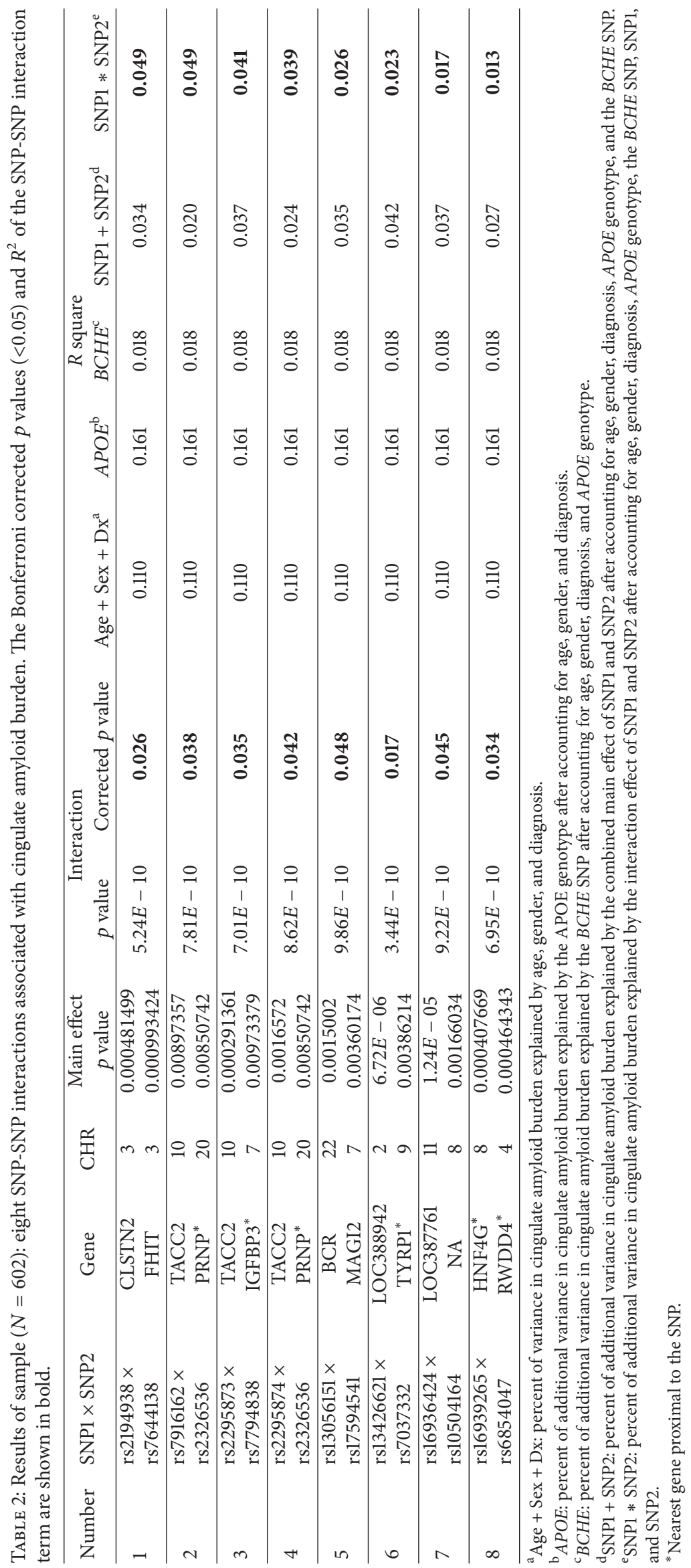




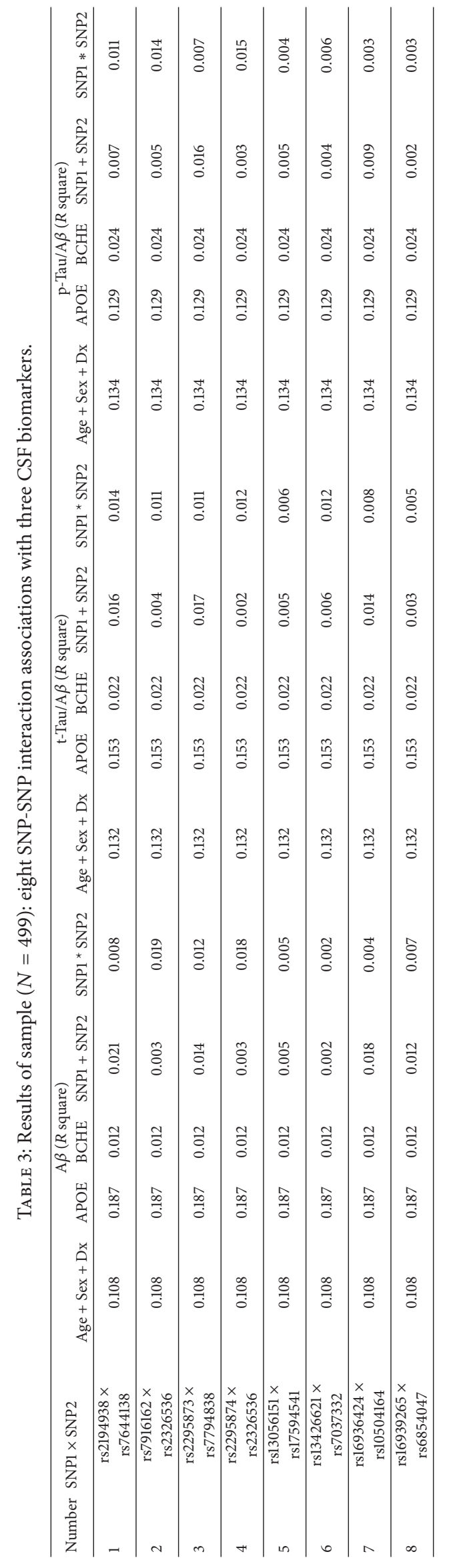




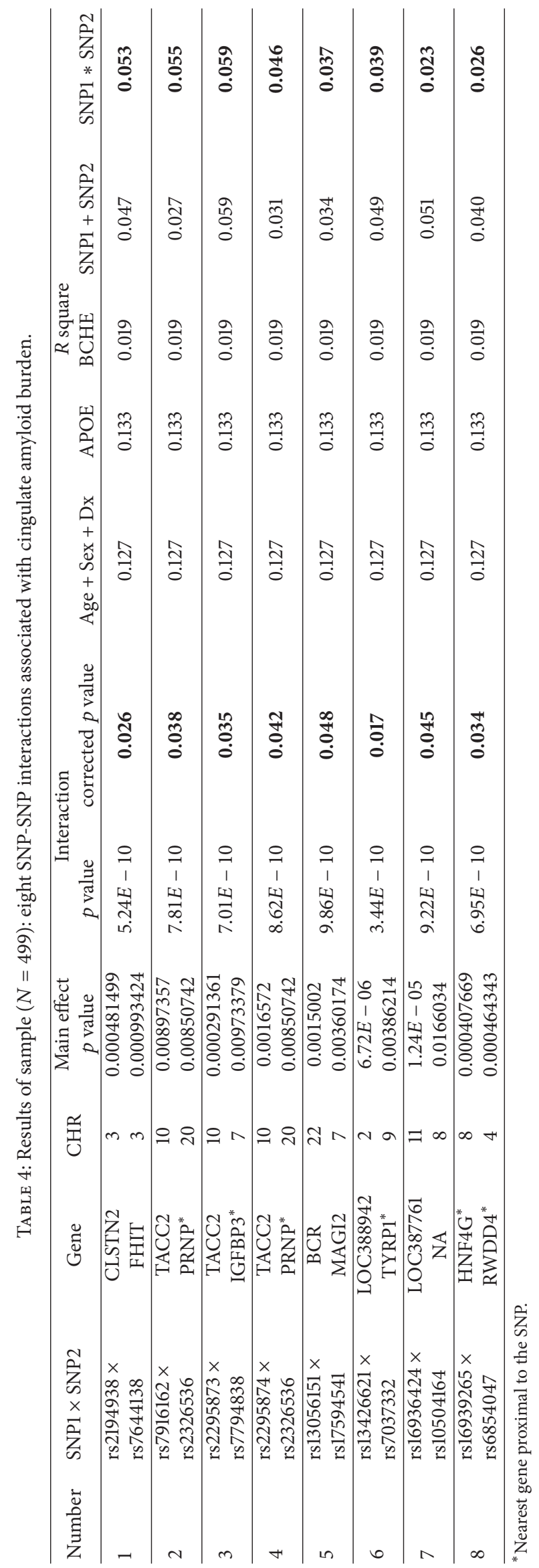




\section{Conclusions}

We performed GWAS and GWIS using amyloid imaging as the quantitative phenotype and investigated the genetic interaction effects on cingulate amyloid-beta $(\mathrm{A} \beta)$ load. The single marker analyses revealed significant hits within or proximal to $A P O E, A P O C 1$, and TOMM40 genes, which were previously implicated in $\mathrm{AD}$. The interaction analyses yielded a few novel interaction findings associated with cingulate amyloid burden, such as those between CLSTN2 and FHIT, between TACC2 and PRNP, between TACC2 and IGFBP 3 , and between BCR and MAGI2. Each of these SNP pairs demonstrated significant interaction effects while their individual main effects were not prominent. This suggests that searching for interaction effects may help solve the problem of missing heritability to some extent. Future studies should attempt to replicate these results in independent datasets with neuroimaging and genetic data, as they become available. Additional pathway analysis and gene sets enrichment analysis could be performed to help understand the genetic interactions between SNPs on amyloid imaging phenotypes and potentially provide critical functional evidence in support of the statistical association findings.

\section{Conflict of Interests}

The authors have no actual or potential conflict of interests including any financial, personal, or other relationships with other people or organizations that could inappropriately influence (bias) their work.

\section{Acknowledgments}

This paper is supported in part by grants from National Natural Science Foundation of China (61471139, 61403092, and 61071174), National Key Scientific Instrument and Equipment Development Projects of China (2012YQ04014001 and 2012YQ04014010), Fundamental Research Funds for the Central Universities (HEUCFT1102, HEUCFT1302, and HEUCFX41303), and the HEU 111 Project at Harbin Engineering University and by NIH R01 LM011360, U01 AG024904, RC2 AG036535, R01 AG19771, P30 AG10133, UL1 TR001108, NSF IIS-1117335, DOD W81XWH-14-2-0151, and NCAA 14132004 at IU. Data collection and sharing for this project were funded by the Alzheimer's Disease Neuroimaging Initiative (ADNI) (National Institutes of Health Grant U01 AG024904) and DOD ADNI (Department of Defense Award no. W81XWH-12-2-0012). ADNI is funded by the National Institute on Aging, the National Institute of Biomedical Imaging and Bioengineering, and through generous contributions from the following: Alzheimer's Association; Alzheimer's Drug Discovery Foundation; Araclon Biotech; BioClinica, Inc.; Biogen Idec Inc.; BristolMyers Squibb Company; Eisai Inc.; Elan Pharmaceuticals, Inc.; Eli Lilly and Company; EuroImmun; F. Hoffmann-La Roche Ltd and its affiliated company Genentech, Inc.; Fujirebio; GE Healthcare; IXICO Ltd.; Janssen Alzheimer Immunotherapy Research \& Development, LLC.; Johnson \& Johnson Pharmaceutical Research \& Development LLC.;
Medpace, Inc.; Merck \& Co., Inc.; Meso Scale Diagnostics, LLC.; NeuroRx Research; Neurotrack Technologies; Novartis Pharmaceuticals Corporation; Pfizer Inc.; Piramal Imaging; Servier; Synarc Inc.; and Takeda Pharmaceutical Company. The Canadian Institutes of Health Research is providing funds to support ADNI clinical sites in Canada. Private sector contributions are facilitated by the Foundation for the National Institutes of Health (http://www.fnih.org/). The grantee organization is the Northern Alzheimer's Disease Cooperative Study at the University of California, San Diego. ADNI data are disseminated by the Laboratory for Neuro Imaging at the University of Southern California. Data used in preparation of this paper were obtained from the Alzheimer's Disease Neuroimaging Initiative (ADNI) database (http://adni.loni.usc.edu/). As such, the investigators within the ADNI contributed to the design and implementation of ADNI and/or provided data but did not participate in analysis or writing of this paper. A complete listing of ADNI investigators can be found at http://adni.loni .usc.edu/wp-content/uploads/how_to_apply/ADNI_Acknowledgement_List.pdf.

\section{References}

[1] D. L. Price and S. S. Sisodia, "Mutant genes in familial Alzheimer's disease and transgenic models," Annual Review of Neuroscience, vol. 21, no. 1, pp. 479-505, 1998.

[2] L. Bertram, C. M. Lill, and R. E. Tanzi, "The genetics of alzheimer disease: back to the future," Neuron, vol. 68, no. 2, pp. 270-281, 2010.

[3] H. Akiyama, K. Ikeda, H. Kondo, M. Kato, and P. L. McGeer, "Microglia express the type 2 plasminogen activator inhibitor in the brain of control subjects and patients with Alzheimer's disease," Neuroscience Letters, vol. 164, no. 1-2, pp. 233-235, 1993.

[4] D. Harold, R. Abraham, P. Hollingworth et al., "Genomewide association study identifies variants at CLU and PICALM associated with Alzheimer's disease," Nature Genetics, vol. 41, pp. 1088-1093, 2009.

[5] O. Belbin, M. M. Carrasquillo, M. Crump et al., "Investigation of 15 of the top candidate genes for late-onset Alzheimer's disease," Human Genetics, vol. 129, no. 3, pp. 273-282, 2011.

[6] M. M. Carrasquillo, T. A. Hunter, L. Ma et al., "Replication of BIN1 association with Alzheimer's disease and evaluation of genetic interactions," Journal of Alzheimer's Disease, vol. 24, no. 4, pp. 751-758, 2011.

[7] P. Hollingworth, D. Harold, R. Sims et al., "Common variants at ABCA7, MS4A6A/MS4A4E, EPHA1, CD33 and CD2AP are associated with Alzheimer's disease," Nature Genetics, vol. 43, no. 5, pp. 429-436, 2011.

[8] A. C. Naj, G. Jun, G. W. Beecham et al., "Common variants at MS4A4/MS4A6E, CD2AP, CD33 and EPHA1 are associated with late-onset Alzheimer's disease," Nature Genetics, vol. 43, no. 5, pp. 436-441, 2011.

[9] H. Shi, O. Belbin, C. Medway et al., "Genetic variants influencing human aging from late-onset Alzheimer's disease (LOAD) genome-wide association studies (GWAS)," Neurobiology of Aging, vol. 33, no. 8, pp. e5-e18, 2012.

[10] J.-C. Lambert, C. A. Ibrahim-Verbaas, D. Harold et al., "Metaanalysis of 74,046 individuals identifies 11 new susceptibility loci for Alzheimer's disease," Nature Genetics, vol. 45, no. 12, pp. 1452-1458, 2013. 
[11] N. F. Bence, R. M. Sampat, and R. R. Kopito, "Impairment of the ubiquitin-proteasome system by protein aggregation," Science, vol. 292, no. 5521, pp. 1552-1555, 2001.

[12] S. G. Potkin, J. A. Turner, G. Guffanti et al., "Genome-wide strategies for discovering genetic influences on cognition and cognitive disorders: methodological considerations," Cognitive Neuropsychiatry, vol. 14, no. 4-5, pp. 391-418, 2009.

[13] B. Dubois, H. H. Feldman, C. Jacova et al., "Research criteria for the diagnosis of Alzheimer's disease: revising the NINCDSADRDA criteria," The Lancet Neurology, vol. 6, no. 8, pp. 734746, 2007.

[14] M. D. Ikonomovic, W. E. Klunk, E. E. Abrahamson et al., "Postmortem correlates of in vivo PiB-PET amyloid imaging in a typical case of Alzheimer's disease," Brain, vol. 131, no. 6, pp. 1630-1645, 2008.

[15] M. S. Albert, S. T. DeKosky, D. Dickson et al., "The diagnosis of mild cognitive impairment due to Alzheimer's disease: recommendations from the National Institute on AgingAlzheimer's Association workgroups on diagnostic guidelines for Alzheimer's disease," Alzheimer's and Dementia, vol. 7, no. 3, pp. 270-279, 2011.

[16] C. M. Clark, J. A. Schneider, B. J. Bedell et al., "Use of florbetapirPET for imaging beta-amyloid pathology," The Journal of the American Medical Association, vol. 305, no. 3, pp. 275-283, 2011.

[17] R. A. Sperling, P. S. Aisen, L. A. Beckett et al., "Toward defining the preclinical stages of Alzheimer's disease: recommendations from the National Institute on Aging-Alzheimer's Association workgroups on diagnostic guidelines for Alzheimer's disease," Alzheimer's \& Dementia, vol. 7, no. 3, pp. 280-292, 2011.

[18] L. Shen, P. M. Thompson, S. G. Potkin et al., "Genetic analysis of quantitative phenotypes in $\mathrm{AD}$ and MCI: imaging, cognition and biomarkers," Brain Imaging and Behavior, vol. 8, no. 2, pp. 183-207, 2014.

[19] S. A. Meda, M. E. I. Koran, J. R. Pryweller, J. N. Vega, and T. A. Thornton-Wells, "Genetic interactions associated with 12-month atrophy in hippocampus and entorhinal cortex in Alzheimer's Disease Neuroimaging Initiative," Neurobiology of Aging, vol. 34, no. 5, pp. 1518.e9-1518.e18, 2013.

[20] I. Mateo, J. L. Vázquez-Higuera, P. Sánchez-Juan et al., "Epistasis between tau phosphorylation regulating genes (CDK5R1 and GSK-3 $\beta$ ) and Alzheimer's disease risk," Acta Neurologica Scandinavica, vol. 120, no. 2, pp. 130-133, 2009.

[21] E. Rodríguez-Rodríguez, I. Mateo, J. Infante et al., "Interaction between HMGCR and ABCA1 cholesterol-related genes modulates Alzheimer's disease risk," Brain Research, vol. 1280, pp. 166-171, 2009.

[22] E. Rodriguez-Rodriguez, J. L. Vázquez-Higuera, P. SánchezJuan et al., "Epistasis between intracellular cholesterol trafficking-related genes (NPC1 and ABCA1) and Alzheimer's disease risk," Journal of Alzheimer's Disease, vol. 21, no. 2, pp. 619-625, 2010.

[23] M. E. I. Koran, T. J. Hohman, and T. A. Thornton-Wells, "Genetic interactions found between calcium channel genes modulate amyloid load measured by positron emission tomography," Human Genetics, vol. 133, no. 1, pp. 85-93, 2014.

[24] M. Thambisetty, Y. An, M. Nalls et al., "Effect of complement CR1 on brain amyloid burden during aging and its modification by APOE genotype," Biological Psychiatry, vol. 73, no. 5, pp. 422428, 2013.

[25] A. J. Saykin, L. Shen, T. M. Foroud et al., "Alzheimer's disease neuroimaging initiative biomarkers as quantitative phenotypes: genetics core aims, progress, and plans," Alzheimer's \& Dementia, vol. 6, no. 3, pp. 265-273, 2010.

[26] L. Shen, S. Kim, S. L. Risacher et al., "Whole genome association study of brain-wide imaging phenotypes for identifying quantitative trait loci in MCI and AD: a study of the ADNI cohort," NeuroImage, vol. 53, no. 3, pp. 1051-1063, 2010.

[27] S. Purcell, B. Neale, K. Todd-Brown et al., "PLINK: a tool set for whole-genome association and population-based linkage analyses," American Journal of Human Genetics, vol. 81, no. 3, pp. 559-575, 2007.

[28] A. L. Price, N. J. Patterson, R. M. Plenge, M. E. Weinblatt, N. A. Shadick, and D. Reich, "Principal components analysis corrects for stratification in genome-wide association studies," Nature Genetics, vol. 38, no. 8, pp. 904-909, 2006.

[29] J. K. Pritchard, M. Stephens, and P. Donnelly, "Inference of population structure using multilocus genotype data," Genetics, vol. 155, no. 2, pp. 945-959, 2000.

[30] K.-L. Huang, K.-J. Lin, I.-T. Hsiao et al., "Regional amyloid deposition in amnestic mild cognitive impairment and Alzheimer's disease evaluated by $\left[{ }^{18} \mathrm{~F}\right] \mathrm{AV}-45$ positron emission tomography in Chinese population," PLOS ONE, vol. 8, no. 3, Article ID e58974, 2013.

[31] W. J. Jagust, S. M. Landau, L. M. Shaw et al., "Relationships between biomarkers in aging and dementia," Neurology, vol. 73, no. 15, pp. 1193-1199, 2009.

[32] L. M. Shaw, H. Vanderstichele, M. Knapik-Czajka et al., "Cerebrospinal fluid biomarker signature in Alzheimer's disease neuroimaging initiative subjects," Annals of Neurology, vol. 65, no. 4, pp. 403-413, 2009.

[33] H. Hampel, K. Blennow, L. M. Shaw, Y. C. Hoessler, H. Zetterberg, and J. Q. Trojanowski, "Total and phosphorylated tau protein as biological markers of Alzheimer's disease," Experimental Gerontology, vol. 45, no. 1, pp. 30-40, 2010.

[34] S. Kim, S. Swaminathan, L. Shen et al., "Genome-wide association study of CSF biomarkers A $\beta 1-42$, t-tau, and p-tau181p in the ADNI cohort," Neurology, vol. 76, no. 1, pp. 69-79, 2011.

[35] C. Herold, M. Steffens, F. F. Brockschmidt, M. P. Baur, and T. Becker, "INTERSNP: genome-wide interaction analysis guided by a priori information," Bioinformatics, vol. 25, no. 24, pp. 3275-3281, 2009.

[36] V. K. Ramanan, S. L. Risacher, K. Nho et al., "APOE and BCHE as modulators of cerebral amyloid deposition: a florbetapir PET genome-wide association study," Molecular Psychiatry, vol. 19, no. 3, pp. 351-357, 2014.

[37] E. H. Corder, A. M. Saunders, W. J. Strittmatter et al., "Gene dose of apolipoprotein E type 4 allele and the risk of Alzheimer's disease in late onset families," Science, vol. 261, no. 5123, pp. 921923, 1993.

[38] R. Guerreiro, J. Brás, A. Wojtas, R. Rademakers, J. Hardy, and N. Graff-Radford, "A nonsense mutation in PRNP associated with clinical Alzheimer's disease," Neurobiology of Aging, vol. 35, no. 11, pp. 2656.e13-2656.e16, 2014.

[39] C. Sassi, R. Guerreiro, R. Gibbs et al., "Investigating the role of rare coding variability in Mendelian dementia genes (APP, PSEN1, PSEN2, GRN, MAPT, and PRNP) in late-onset Alzheimer's disease," Neurobiology of Aging, vol. 35, no. 12, pp. 2881.e1-2881.e6, 2014.

[40] M. Ikonen, B. Liu, Y. Hashimoto et al., "Interaction between the Alzheimer's survival peptide humanin and insulin-like growth factor-binding protein 3 regulates cell survival and apoptosis," Proceedings of the National Academy of Sciences of the United States of America, vol. 100, no. 22, pp. 13042-13047, 2003. 
[41] P. Kochunov, J. Charlesworth, A. Winkler et al., "Transcriptomics of cortical gray matter thickness decline during normal aging," NeuroImage, vol. 82, pp. 273-283, 2013.

[42] S. G. Potkin, G. Guffanti, A. Lakatos et al., "Hippocampal atrophy as a quantitative trait in a genome-wide association study identifying novel susceptibility genes for Alzheimer's disease," PLoS ONE, vol. 4, no. 8, Article ID e6501, 2009.

[43] M. W. Weiner, D. P. Veitch, P. S. Aisen et al., "The Alzheimer's disease neuroimaging initiative: a review of papers published since its inception," Alzheimer's and Dementia, vol. 9, no. 5, pp. el11-e194, 2013. 

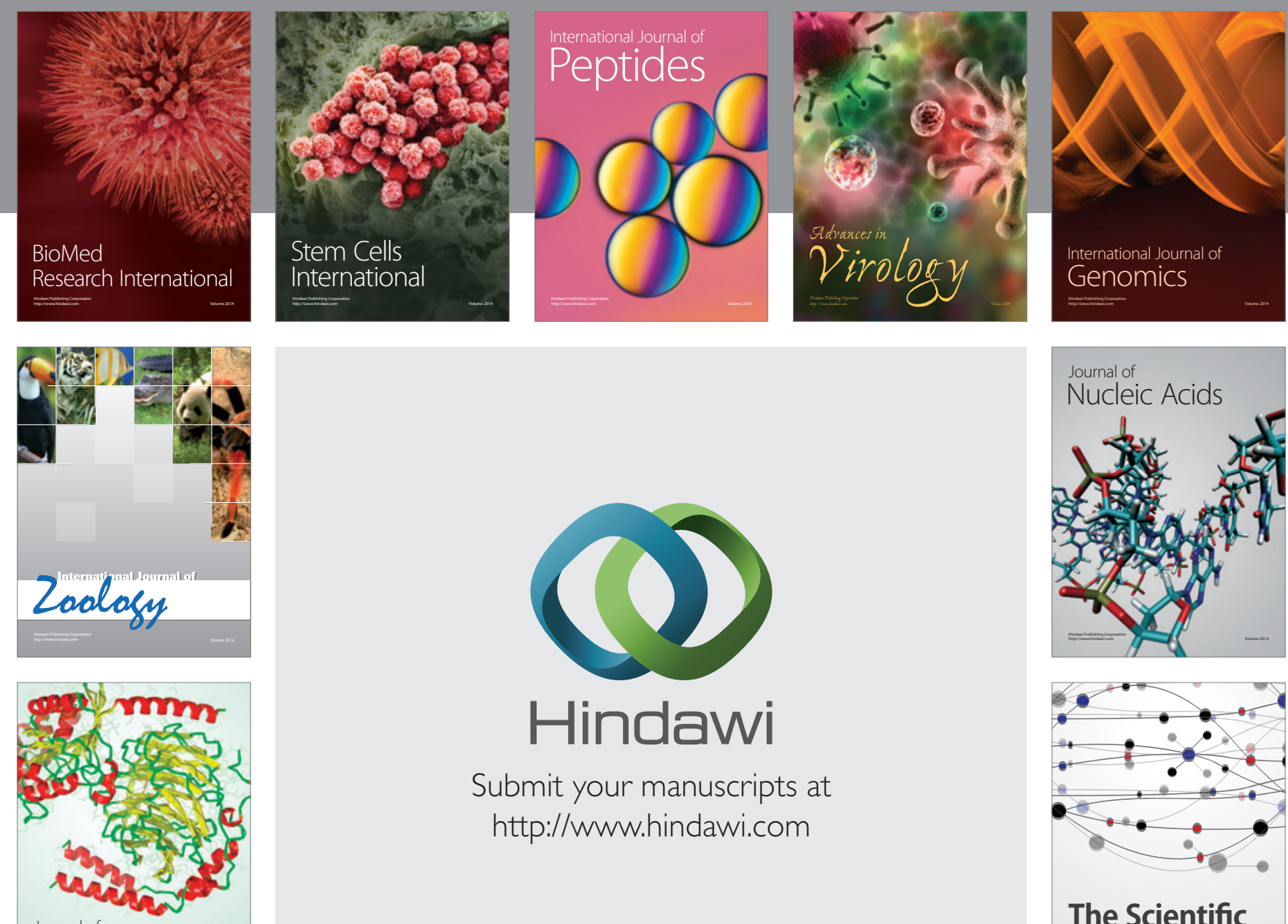

Submit your manuscripts at

http://www.hindawi.com

Journal of
Signal Transduction
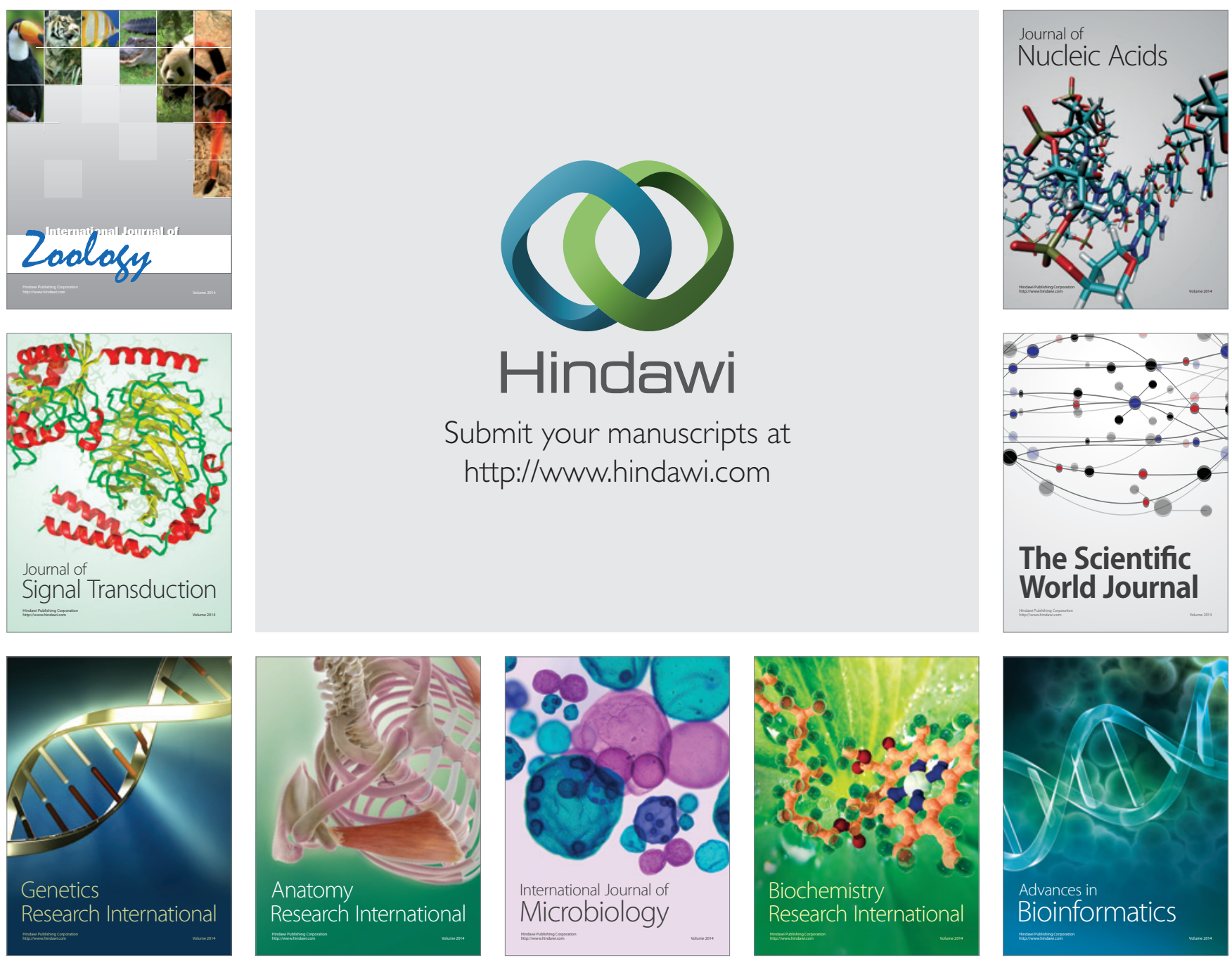

The Scientific World Journal
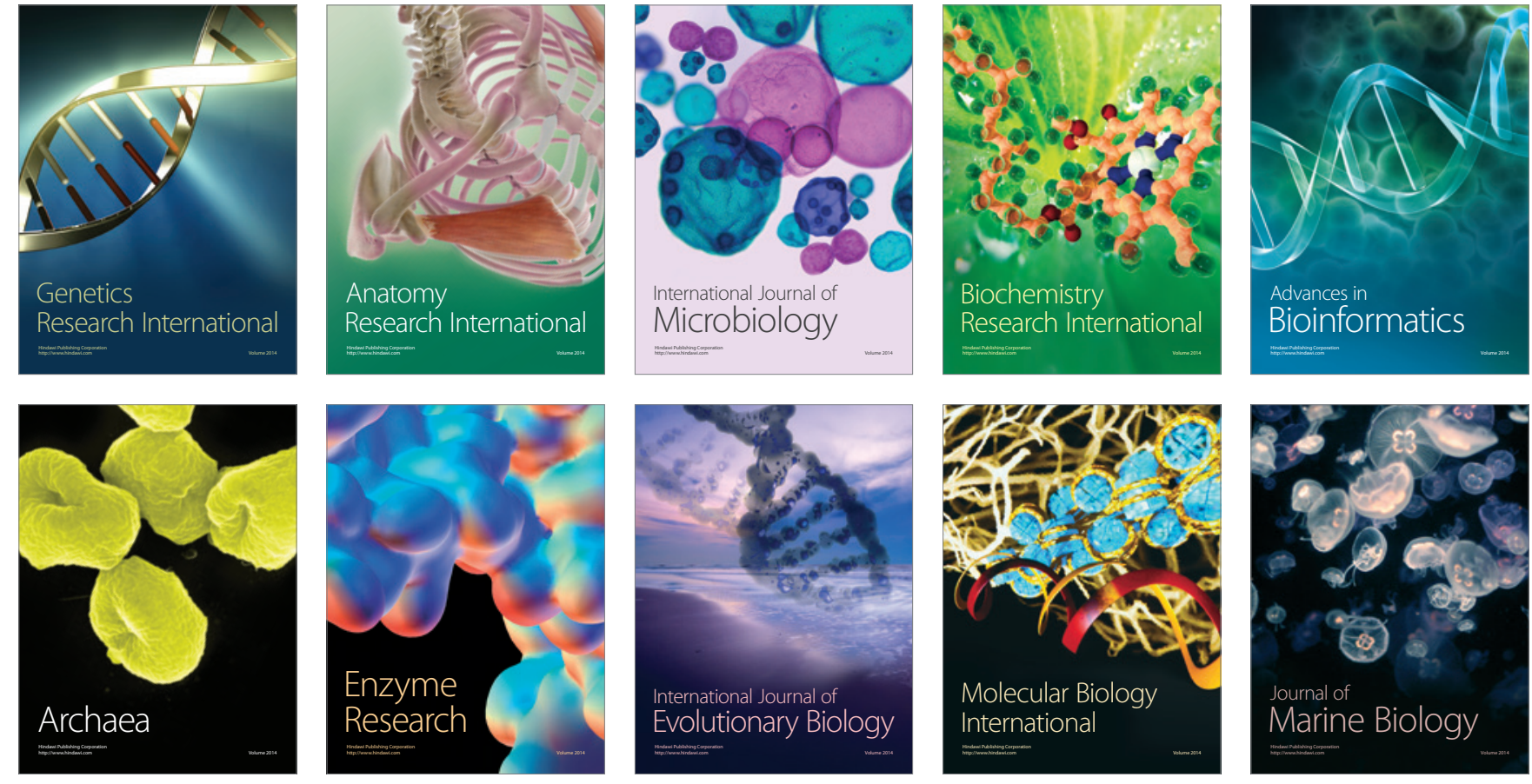